\section{Comment on "Why Are There So Many Species of Herbivorous Insects in Tropical Rainforests?"}

David A. Norton ${ }^{1}$ and Raphael K. Didham ${ }^{2 *}$

Novotny et al. (Reports, 25 August 2006, p. 1115) argued that higher herbivore diversity in tropical forests results from greater phylogenetic diversity of host plants, not from higher host specificity. However, if host specificity is related to host abundance, differences in relative host abundance between tropical and temperate regions may limit any general conclusion that herbivore diversity scales directly with host-plant diversity.

$\mathrm{N}$ ovotny et al. (1) concluded that once the confounding effects of sampling intensity and the comparative phylogenetic diversity of host plants have been controlled for, there are no significant differences in either herbivore species density or host specificity of herbivores on tropical versus temperate tree species. Instead, they posited that higher herbivore species diversity in tropical forests must result from greater phylogenetic diversity of tropical vegetation - a classic "diversity begets diversity" argument (2). We suggest that differences in host-plant abundance between tropical and temperate regions may limit any general conclusion that herbivore diversity scales directly with host-plant diversity.

We agree that host plant diversity is a critically important determinant of tropical herbivore diversity. However, any strict interpretation by Novotny et al. that host specificity is constant

${ }^{1}$ School of Forestry, University of Canterbury, Private Bag 4800, Christchurch, New Zealand. ${ }^{2}$ School of Biological Sciences, University of Canterbury, Private Bag 4800, Christchurch, New Zealand.

*To whom correspondence should be addressed. E-mail: raphael.didham@canterbury.ac.nz
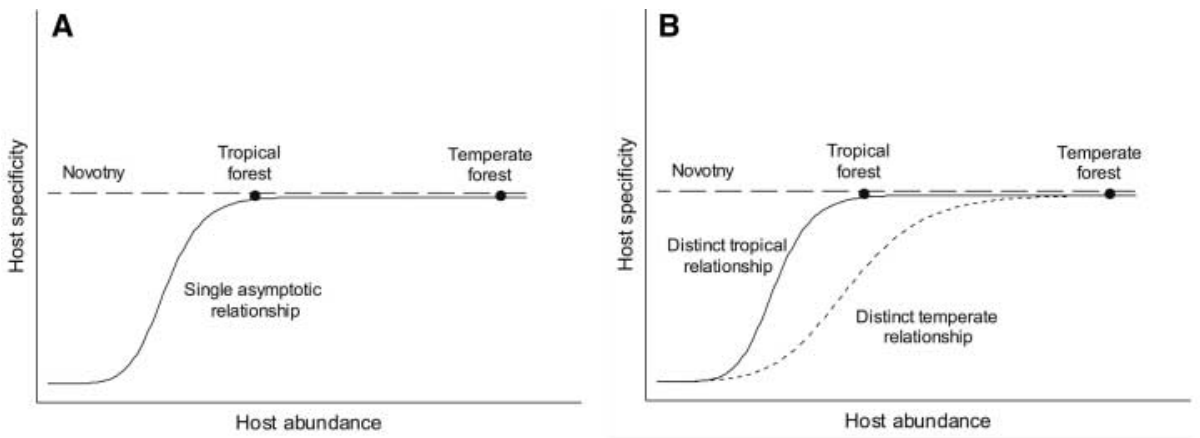

Fig. 1. Host plant abundance may impose an upper limit on the diversity of host-dependent species. Novotny et al. found that host specificity of herbivore species is constant on commonly occurring tropical and temperate tree species (dashed line), but this does not take into account a possible relationship between host abundance and host specificity. Two hypothetical alternative possibilities for this relationship are (A) a single asymptotic abundance-

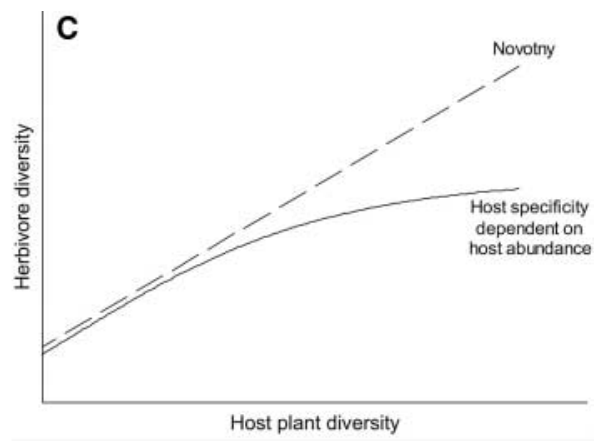

densities and constant host specificity rates to all host plant species, in general, may not be warranted without explicit consideration of relative host abundance.

The question is whether the theoretical rationale for expecting a positive relationship between host abundance and specificity has an important bearing on empirical host specificity relationships across regions. Unfortunately, there are no empirical data available on the form of the host abundance versus specificity relationship for herbivorous insects. However, there are at least two alternative interpretations of the equivalence in tropical and temperate host specificity data presented by Novotny et al. (1), and these have important implications for the apparent scaling of herbivore diversity with host plant diversity. First, the unresolved relationship between host abundance and specificity may have a strict lower limit arising from evolutionary constraints on host specialization, but approach essentially the same value in both temperate and tropical herbivore communities (Fig. 1A). Alternatively, the slope of the relationship between host abundance and specificity may differ substantially between biomes, such that the observed equivalence in host specificity in Novotny et al. may be coincidental (Fig. 1B). The latter would imply that host specificity may actually be higher in the tropics for an equivalent degree of host abundance, which would be an exciting proposition to test. In both of these hypothetical alternatives, there will be a net difference in host specificity between tropical and temperate forests, whether this is due simply to a quantitative difference in the diversity of rare host species in the tropics or to a qualitative difference in the abundance-specificity relationship. Therefore, although we agree that host plant diversity is indeed a critically important determinant of tropical herbivore diversity, it is unlikely that herbivore diversity will scale proportionately with increasing host diversity (Fig. 1C), but rather host plant abundance may

specificity relationship (solid line), or (B) distinct abundance-specificity relationships for tropical (solid line) versus temperate (dotted line) regions. (C) In contrast to Novotny et al. (dashed line), we argue that both of these hypothetical alternatives $[(A)$ and $(B)]$ imply that host abundance will impose an upper limit on the scaling relation between herbivore diversity and host diversity (solid line) because the majority of host tree species in the tropics are rare. 
impose an upper limit on the diversity of hostdependent species.

\section{References}

1. V. Novotny et al., Science 313, 1115 (2006).

2. N. Janz, S. Nylin, N. Wahlberg, BMC Evol. Biol. 6, 4 (2006).

3. C. K. Kelly, T. R. E. Southwood, Proc. Natl. Acad. Sci. U.S.A. 96, 8013 (1999).
4. R. A. Beaver, Nature 281, 139 (1979).

5. D. H. Janzen, Ecology 62, 532 (1981).

6. A. F. G. Dixon, P. Kindlmann, ]. Leps, ]. Holman, Am. Nat. 129, 580 (1987).

7. B. A. Hawkins, M. R. Shaw, R. R. Askew, Am. Nat. 139, 58 (1992).

8. D. A. Norton, P. ]. de Lange, Funct. Ecol. 13, 552 (1999).

9. D. A. Norton, M. A. Carpenter, Trends Ecol. Evol. 13, 101 (1998)
10. D. P. Vazquez, R. Poulin, B. R. Krasnov, G. I. Shenbrot, ]. Anim. Ecol. 74, 946 (2005).

11. J. Jaenike, Annu. Rev. Ecol. Syst. 21, 243 (1990).

12. ]. N. Thompson, The Coevolutionary Process (University of Chicago Press, Chicago, 1994).

13. V. Novotny et al., Nature 416, 841 (2002).

7 November 2006; accepted 22 February 2007 10.1126/science. 1137249 